\title{
Face and Gesture-Based Interaction for Displaying Comic Books
}

\author{
Hang-Bong Kang and Myung-Ho Ju \\ Dept. of Computer Eng. Catholic University of Korea \\ \#43-1 Yokkok 2-dong Wonmi-Gu, Bucheon, Gyonggi-Do Korea \\ hbkang@catholic.ac.kr
}

\begin{abstract}
In this paper, we present human robot interaction techniques such as face pose and hand gesture for efficient viewing comics through the robot. For the controlling of the viewing order of the panel, we propose a robust face pose recognition method using the pose appearance manifold. We represent each pose of a person's face as connected low-dimensional appearance manifolds which are approximated by the affine plane. Then, face pose recognition is performed by computing the minimal distance from the given face image to the sub-pose manifold. To handle partially occluded faces, we generate an occlusion mask and then put the lower weights on the occluded pixels of the given image to recognize occluded face pose. For illumination variations in the face, we perform coarse normalization on skin regions using histogram equalization. To recognize hand gestures, we compute the center of gravity of the hand using skeleton algorithm and count the number of active fingers. Also, we detect index finger's moving direction. The contents in the panel are represented by the scene graph and can be updated according to the user's control. Based on the face pose and hand gesture recognition result, an audience can manipulate contents and finally appreciate the comics in his own style.
\end{abstract}

Keywords: Face pose recognition, Hand gesture recognition, Human robot interaction.

\section{Introduction}

Recently, various intelligent robots are developed and used in the range of application from the industrial manufacturing environment to human environment for service and entertainment. Since robots for entertainment are new media with mobility to display various contents to audiences, human robot interaction (HRI) plays an important role in displaying various contents through robots. For example, children can read and hear fairy tales, comics and songs from the robots. However, the traditional contents display method through the robots is usually linear and limited. For efficient viewing of comics, in particular, it is desirable for the user to control the viewing order of panels in comics and manipulate objects in the specified panel. To effectively interact with intelligent robots, it is desirable for a robot to recognize a user's face pose and hand gestures. 
There have been some research works on face pose and hand gesture recognition. For face pose recognition approaches, Pentland et al. [1] proposed view-based eigenspace approach to deal with various face appearances. Moghaddam et al. [2], [3] also suggested various probabilistic visual learning methods for face recognition. Lee et al. [4], [5] presented video-based face recognition using probabilistic appearance manifolds. Their method showed good results in face recognition, but have some limitations to estimate robust face poses in natural environments. It is because the face pose detection is very difficult due to occlusion and illumination changes in face. $\mathrm{Ju}$ and Kang [6] proposed a robust face pose recognition method that works even with partial occlusion or illumination variations for human robot interaction. For hand gesture recognition approaches, Davis and Shah [7] used markers on the finger tips. By detecting the presence and color of the markers, active fingers in the gesture are identified. Chang et al. [8] used a curvature space method for finding the boundary contours of the hand. This approach is robust but requires large computing time. Hasanuzzaman et al. [9] used subspace method to recognize face and hand gesture for human robot interaction. The accuracy of their system depends on the accuracy of the pose detection results. Malima et al. [10] proposed a fast algorithm to recognize a limited set of hand gestures for human robot interaction. Their method is invariant to translation, rotation and scale of the hand, but has problems in precise hand segmentation.

In this paper, we propose a new human robot interaction system for viewing comics using video-based face pose and hand gesture recognition. Fig. 1 shows our scheme for HRI. The input image is taken from the camera on the top of the robot and skin-like regions are extracted. Then, we use morphological filters to filter noise and holes. Face detection and hand segmentation are executed from probable face and hand regions. After that, face pose recognition is performed to control the viewing order of the comics and hand gesture recognition is performed to manipulate objects in the activated panel. The remainder of the paper is organized as follows. Section 2 discusses face pose appearance manifold and face pose recognition method in the cases of partial occlusion and illumination variations. Section 3 presents our hand gesture recognition method. Section 4 shows our human robot interaction system for displaying comic books. Section 5 presents experimental results of our proposed method.

\section{Video-Based Face Pose Recognition}

In this section, we will discuss face pose estimation method using face appearance manifold. We also deal with two cases of partial occlusion and illumination variations for robust control of robots.

\subsection{Video-Based Face Pose Recognition}

For a given face image, its dimensionality is equal to the number of pixels $\mathrm{D}$ in the image. If the face surface is smooth, its appearance can be constrained and confined to an embedded face manifold of dimension $d<<D$ as in [3]. We represent face pose appearance manifold by a set of simple linear sub-pose manifolds using Principal Component of Analysis (PCA). From the face pose manifold $P^{n}$, the face pose 
recognition task is to find sub-pose $n^{*}$ by computing the minimal distance from the given face image $I$ to sub-pose manifold such as

$$
n^{*}=\arg \min _{n} d^{2}\left(I, P^{n}\right)
$$

Since the distance can be represented as conditional probability $p\left(P^{n} \mid I\right)$ as in [5], Eq. (1) is

$$
n^{*}=\arg \max _{n} p\left(P^{n} \mid I\right)
$$

where $p\left(P^{n} \mid I\right)=\frac{1}{\Lambda} \exp \left(\frac{-1}{\sigma^{2}} d^{2}\left(I, P^{n}\right)\right)$, and $\Lambda$ is the normalization term.

The continuous pose estimation in the video-based face recognition framework is to estimate the current sub-pose manifold $P_{t}^{n}$ given current face image $I_{t}$ and the previous sub-pose $p_{t-1}^{m}$.

$$
\begin{aligned}
P_{t}^{n^{*}} & =\arg \max _{n} p\left(P_{t}^{n} \mid I_{t}, P_{t-1}^{m}\right) \\
& =\arg \max _{n} \frac{1}{\Lambda} p\left(I_{t} \mid P_{t}^{n}, P_{t-1}^{m}\right) p\left(P_{t}^{n} \mid P_{t-1}^{m}\right) \\
& =\arg \max _{n} \frac{1}{\Lambda} p\left(I_{t} \mid P_{t}^{n}\right) p\left(P_{t}^{n} \mid P_{t-1}^{m}\right)
\end{aligned}
$$

where $\Lambda$ is the normalization term, the image $I_{t}$ and $P_{t-1}^{m}$ are independent.

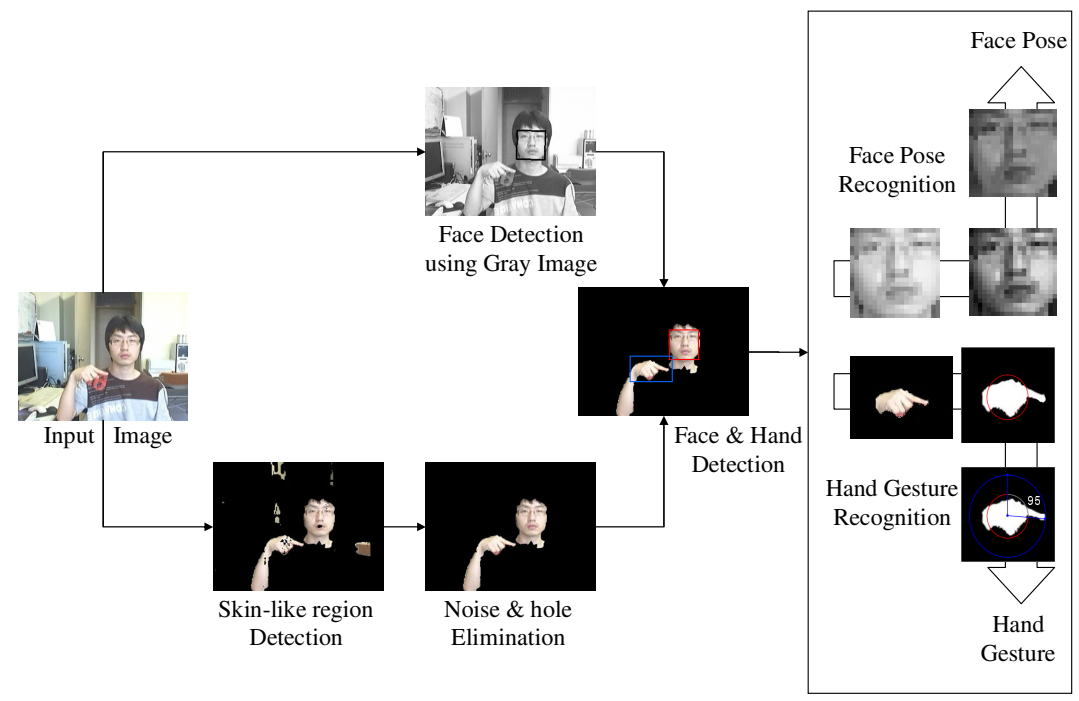

Fig. 1. Our proposed face pose and hand gesture recognition scheme 
The likelihood probability $p\left(I_{t} \mid P_{t}^{n}\right)$ can be estimated using eigenspace decomposition as in [3]. Using PCA, principal component feature vector $y=\Phi_{M}^{T} \tilde{I}$ is obtained, where $\Phi_{M}^{T}$ refers to the submatrix of $\Phi$ containing the principal eigenvectors and $\tilde{I}=I-\bar{I}$ refers to the mean-normalized image vector. If we assume a Gaussian distribution, the likelihood probability can be represented by the product of two Gaussian densities [3]. In other words,

$$
p\left(I_{t} \mid P_{t}^{n}\right)=\left[\frac{\exp \left(-\frac{1}{2}\left(\sum_{i=1}^{M} \frac{y_{i}^{2}}{\lambda_{i}}\right)\right)}{(2 \pi)^{\frac{M}{2}} \prod_{i=1}^{M} \lambda_{i}^{1 / 2}}\right]\left[\frac{\exp \left(-\frac{\varepsilon^{2}\left(I_{t}\right)}{2 \rho}\right)}{(2 \pi \rho)^{(N-M) / 2}}\right]
$$

where $\mathrm{N}$ denotes the dimension of the image space, $\mathrm{M}$ denotes the dimension of subpose space, $\lambda$ denotes eigenvalue, $\varepsilon^{2}\left(I_{t}\right)=\sum_{i=M+1}^{N} y_{i}^{2}$ is the residual reconstruction error, and $\rho=\frac{1}{N-M} \sum_{i=M+1}^{N} \lambda_{i}$.

The transition probability between sub-poses $p\left(P^{i} \mid P^{j}\right)$ in Eq. (3) represents the temporal dynamics of the face movement in the training sequence [5]. When two subposes of face are not connected, the transition probability is 0 .

\subsection{Handling of Partially Occluded Face}

For the robust face pose recognition, it is necessary to handle partially occluded faces by hands or other stuffs [6]. The intensity of an occluded pixel is different from that of the corresponding pixel in the training pose data. In recognizing face pose of the given image, we generate an occlusion mask in which the value of each pixel represents the degree of occlusion of that pixel. According to the occlusion mask, we put lower weights on occluded pixels when computing the distance like $d^{2}\left(I, P^{n}\right)$ in Eq. (1). At start, initial face data is assumed as the front pose and an occlusion mask is constructed from the initial image and the front face pose of training data. After that, the occlusion mask is used to recognize the pose of the next face data. This is shown in Fig. 2.

To compute the degree of occlusion $O D_{i}$, we define intensity difference $I D_{i}$ at pixel $i$ as

$$
I D_{i}=\left\|I_{i}-E_{i}\right\|
$$

To normalize or balance the intensity difference at each pixel, Eq. (5) becomes

$$
I D_{i}=\sqrt{\left(\frac{I_{i}-\mu_{i}}{\sigma_{i}}\right)^{2}}
$$


where $I_{i}$ is the intensity value at pixel $i, \mu_{i}$ and $\sigma_{i}$ are pixel $i$ 's mean value and variance in the training data, respectively. If the pixel's intensity difference is larger than the threshold value, it will be determined as an occluded pixel. If we assume that the distribution of $I D_{i}$ is Gaussian distribution, the degree of occlusion of the $i^{\text {th }}$ pixel $O D_{i}$ is computed as

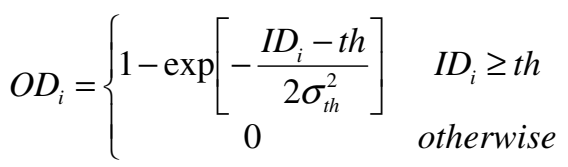

where $\sigma^{2}{ }_{t h}$ is the variance of the pixel differences less than threshold values. To determine the threshold value $t h$ in Eq. (7), we compute the histogram of $I D_{i}$ from the sample data of the sub-pose manifold, and the point of $95 \%$ in the accumulated histogram is selected as a threshold value.

\subsection{Pose Recognition of Face Data with Illumination Variations}

Handling illumination changes is another important factor for robust face pose recognition. Under practical imaging conditions, the image differences due to changing illumination may be critical in the face pose estimation. In addition, if preprocessing is not performed on the face data with illumination variations, the recognition results will be poor because they do not capture non-linear variation in face pose appearance due to illumination changes.

Similar to Arandjelovic et al. [11], we remove the background from the bounding box of a detected face by set-specific skin color segmentation and then normalize for global illumination changes by histogram equalization. The removal of background is helpful in face pose recognition because only face appearance information is used in computing the probabilistic distance like Eq. (2).

\section{Hand Gesture Recognition}

In this section, we present our hand gesture recognition method. Our aim is to recognize a small set of hand gesture commands in real time. We first segment skinlike regions based on skin color statistics and remove false hand candidates using size constraint. Then, we detect precise hand regions and compute center of gravity (COG) of the hand region. After that, we extract the farthest point and the nearest point from COG. Based on these features, we count the number of activated fingers in the hand.

\subsection{Hand Segmentation}

To detect hand candidates, we find the pixels that are likely to skin-like regions. $\mathrm{YCbCr}$ color representation system is used for skin-like region segmentation because it provides an effective use of chrominance information for modeling the human skin color. The RGB image which is taken from the video camera is converted to $\mathrm{YCbCr}$ 
color space and skin area are determined by the skin color range. Fig. 3 shows $\mathrm{YCbCr}$ distributions of a sample of skin region. Chrominance components $\mathrm{Cb}$ and $\mathrm{Cr}$ play important roles in detecting skin-like regions from the color image.

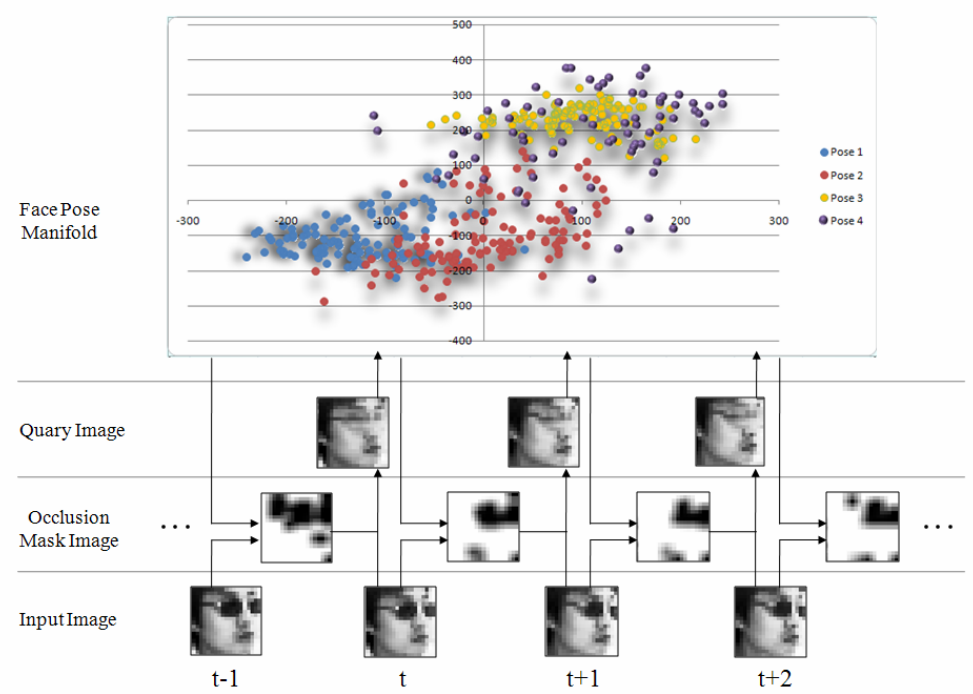

Fig. 2. Pose recognition from the partially occluded face

We extract three large connected skin-like regions and remove false positives using size constraints. Noise and holes are filtered by morphological dilation and erosion operations. From the three large regions, we remove the face region that is detected from gray image using Viola and Jones' method [13]. The remaining regions are the probable hand regions.

\subsection{Hand Gesture Recognition}

To recognize the hand gesture, it is necessary to segment hand regions precisely from the probable hand regions because the forearm features do not represent important information about hand pose. Fig. 4 shows probable COG of hand regions. We first compute the skeleton of the hand regions and then find a circle to cover the hand region along the skeleton axis. The circle will be small at the wrist point and will be large in the hand region. When we find the largest circle covering hand region along the skeleton axis like Fig. 4, the center of that circle is assigned as COG of the hand. And the hand regions are segmented at the wrist point.

From the COG, we compute the distances between the most extreme points such as the farthest point and the nearest point in the hand to the COG. This is shown in Fig. 5. Usually the farthest point is the tip of the longest active finger in the particular gesture. To count the number of active fingers, we draw a circle on the COG like in [10]. The radius of the circle is 0.7 of the farthest distance which is from the COG to the farthest point. This is shown in Fig. 6. After that, we can count the intersection 


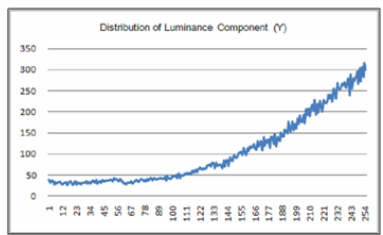

Y

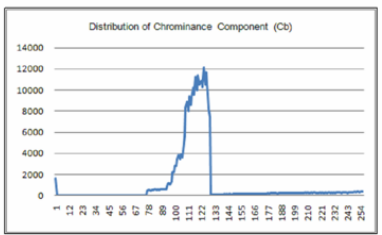

$\mathrm{C}_{\mathrm{b}}$

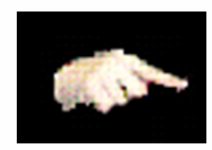

Sample skin region

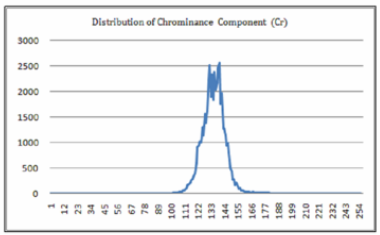

$\mathrm{C}_{\mathrm{r}}$

Fig. 3. YCbCr distribution for the sample skin region

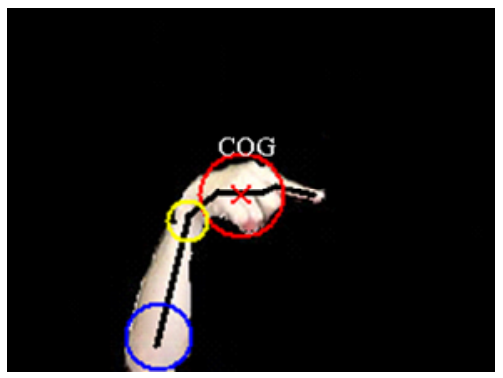

Fig. 4. COG of the hand

area for counting active fingers in the hand. To detect the fist of the hand, we compute the relationship between the nearest distance and the farthest distance. If the relationship like Eq. (8) is satisfied, we classify that the hand gesture is fist.

$$
\text { Farthest_dist }<1.7 * \text { Nearest_dist }
$$

We can classify hand gestures into the fist and the number of active fingers. These classifications are used in the control of object manipulation in the activated panel of the comics.

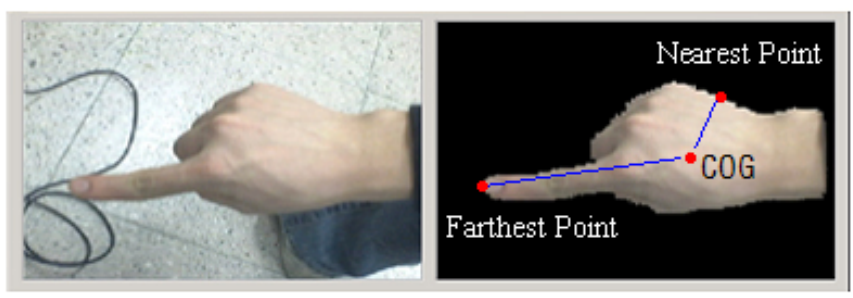

Fig. 5. The farthest point, the nearest point and COG 


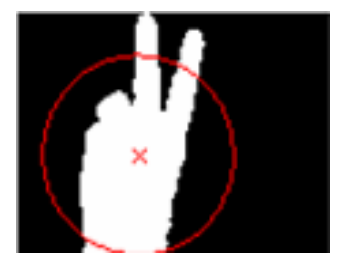

Fig. 6. Two active fingers: "Count 2"

\section{Face Pose and Hand Gesture Based Human Robot Interaction}

To control the viewing style of the comics from the wheeled robot, we use the face pose and hand gesture. In this section, we present human robot interaction methods in viewing comics using face pose and hand gesture.

\subsection{Face Pose and Hand Gesture-Based Control}

A user appreciates comics from the wheeled robot equipped with a web cam like ER1[12]. Fig. 7 shows our scheme. The input image is taken from the camera and skin-like regions are extracted as stated in Section 3. Then, we use morphological filters to filter noise and holes. Face detection and hand segmentation are executed from probable face and hand regions. A Face candidate is detected from the gray image using Viola and Jones' method [13]. Hand segmentation is performed by finding a large circle along the skeleton of probable hand regions as stated in Section 3. After that, face pose recognition is performed to control the viewing order of the comics and hand gesture recognition is performed to manipulate objects in the activated panel.

The face pose controls the activation of panels in comics into four directions such as up, down, right and left panels. Hand gesture controls the activated panel by "zoom in" or "zoom out" using "fist" and "count 5". When the panel is zoomed in, the robot moves toward to the user about $20 \mathrm{~cm}$ and then a user can move object such as move up, down, left and right. Translation of the activated object is performed by index finger's direction like Fig. 8. Scaling is performed by "count 3" and "count 4". Rotation is performed using two hands consisting of "fist" and "count 5". When the object is rotated, the robot also makes a circle. According to the user's various manipulations, new object-based comic contents are created.

\subsection{Scene Graph-Based Object Representation}

The scene in the panel of the comics is represented by the scene graph. Fig. 9 shows an example of scene graph which is simplified version used in VRML97 [14]. Each leaf node in the scene graph has information about location, rotation, and scale of the object. According to the user's hand gesture, the activated object is transformed by translation, rotation or scaling. The modified scene is maintained using the update of scene graph like Fig. 9(b). The attributes in each node have two values: one is original value, and the other one is current value. By maintaining the scene graph, we can recover original panel of the comics easily. 


\section{Experimental Results}

We experimented our face pose and hand gesture driven comics viewing method on a wheeled robot ER1. Fig. 10 shows an example of human robot interaction using face pose and hand gesture for viewing comics. The wheeled robot's camera images are transmitted to the computer and the commands for viewing order of comics and robot's movement are computed from the face pose and hand gesture recognition results.

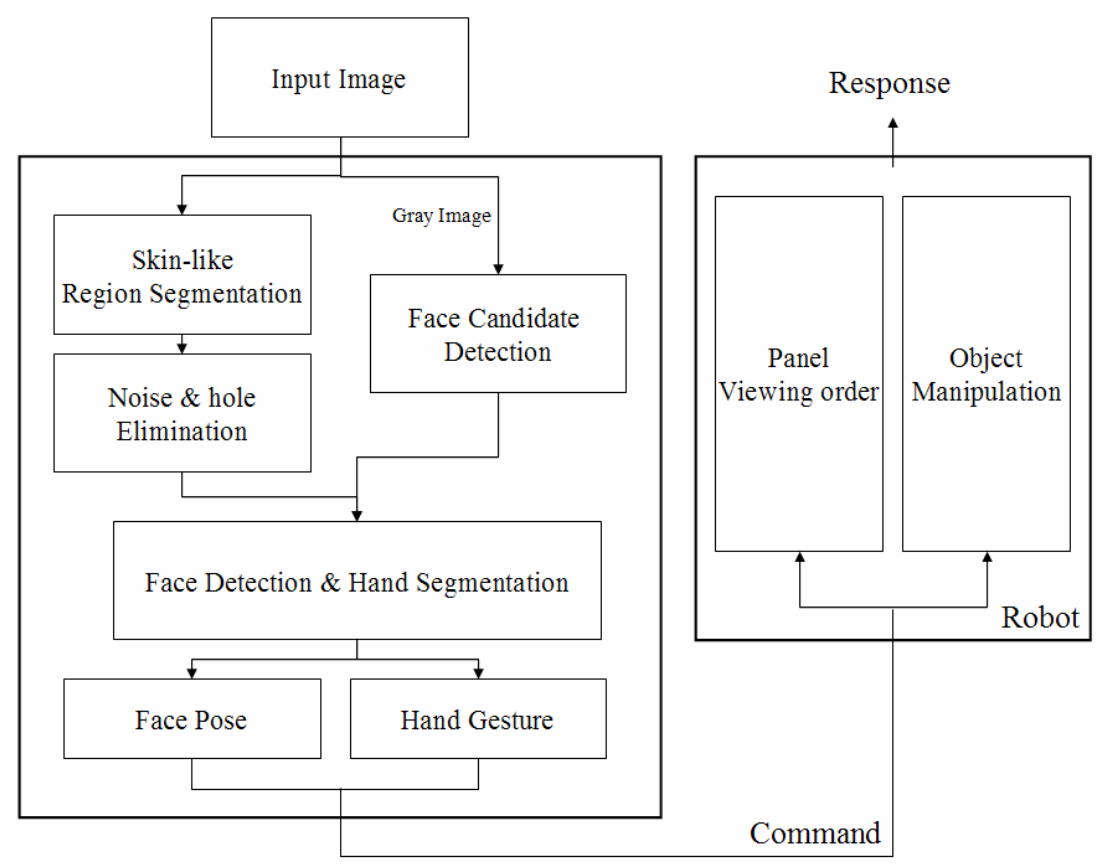

Fig. 7. Our proposed face pose and hand gesture recognition

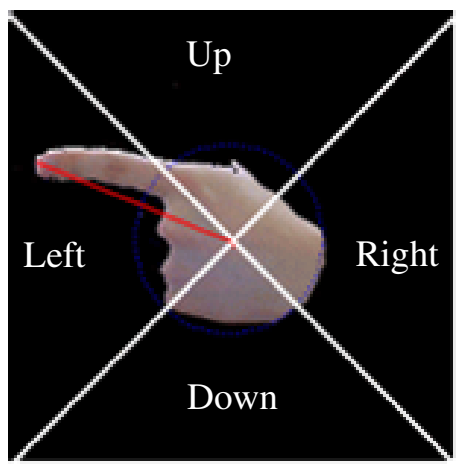

Fig. 8. Four translation directions using an index finger 

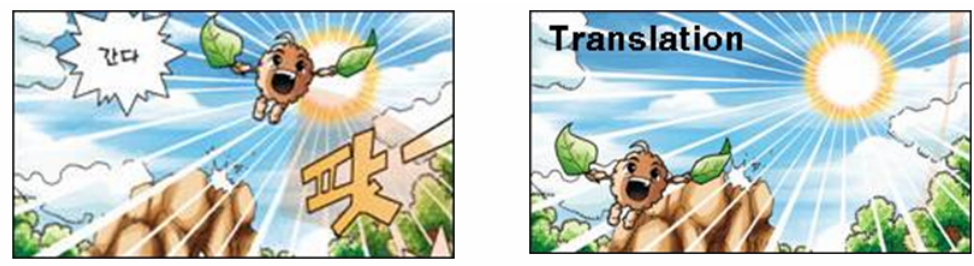

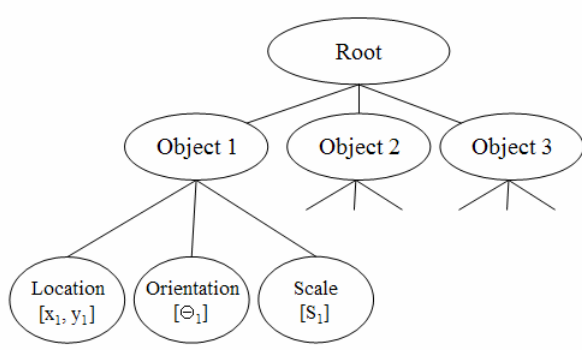

(a)

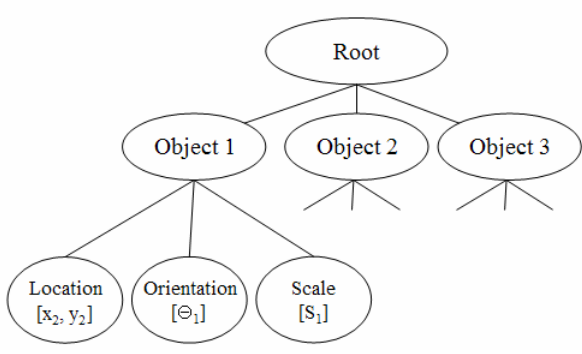

(b)

Fig. 9. Scene Graph. (a) original, (b) updated scene graph according to translation.

To test face pose recognition, 12 image sequences are captured from 6 subjects using web camera on the robot. The image resolution is converted to $320 \times 240$ pixels. The duration of each sequence is about 40 seconds and the frame rate is 15 frames per second. For pose appearance manifold learning, we picked 2 sequences from each person for training sequences and cropped face images. The face images are normalized to 20x 20 pixels. Then, we construct 5 sub-pose manifolds from cropped images using PCA. For each manifold, the dimension of sub pose $M$ in Eq. (4) is set to 20 .

To control the viewing order with the face pose information, the user's face should be first detected and then recognized. After that, the face tracking process is executed for video-based face pose recognition. For face tracking, 80 sample windows having different sizes and orientations are created around the previous face position. Each sample window is converted to 20 x 20 size window and skin regions are extracted. For the skin regions, histogram is equalized. Then, the sample windows are compared with the identified person's face sub-pose manifold. At this time, the face sub-pose manifold is the face pose of the previous frame. If the minimal distance between the sample window and the sub-pose appearance manifold is larger than the threshold value, we conclude the tracked face is a wrong one and a new face detection process is executed. Otherwise, the face sample window is determined to be a correct one and current face pose is estimated using Eq. (3). We experimented face pose recognition on 10 sequences captured from 6 persons. Table 1 shows our face pose recognition results. Our proposed method achieves $93.8 \%$ face pose recognition rate on average. 


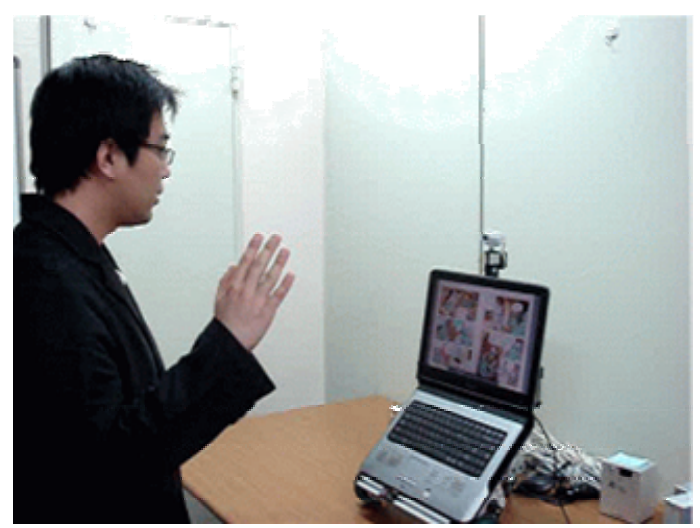

Fig. 10. Face pose and hand gesture based human robot interaction

Table 1. Pose estimation results

\begin{tabular}{ccc}
\hline $\begin{array}{c}\text { Pose (Total } \\
\text { Frame No.) }\end{array}$ & $\begin{array}{c}\text { Correct } \\
\text { Frame No. }\end{array}$ & $\begin{array}{l}\text { Proposed } \\
\text { method }\end{array}$ \\
\hline $0(560)$ & 527 & $94.11 \%$ \\
$1(1,263)$ & 1,202 & $95.17 \%$ \\
$2(575)$ & 570 & $99.13 \%$ \\
$3(331)$ & 287 & $83.99 \%$ \\
$4(430)$ & 387 & $90.00 \%$ \\
Total $(3,159)$ & 2,964 & $93.83 \%$ \\
\hline
\end{tabular}

Table 2. Face pose recognition results for partially occluded faces

\begin{tabular}{ccc}
\hline Pose & $\begin{array}{c}\text { Proposed } \\
\text { method }\end{array}$ & Non-mask \\
\hline 0 & $92.98 \%$ & $81.02 \%$ \\
1 & $93.32 \%$ & $84.42 \%$ \\
2 & $93.98 \%$ & $80.66 \%$ \\
3 & $69.33 \%$ & $68.48 \%$ \\
4 & $93.92 \%$ & $88.63 \%$ \\
Total & $92.11 \%$ & $82.85 \%$ \\
\hline
\end{tabular}

We also tested our face pose recognition from partially occluded faces. The occlusion mask like Fig. 2 is generated by detecting occluding pixels. The face pose recognition results are shown in Table 2. Our method shows desirable performance like $92.11 \%$. For faces under various illumination variations, our face pose recognition results are shown in Table 3. Our proposed method improves the recognition rate in comparison with the non-histogram equalization method. 
Table 3. Face pose recognition results for illumination changed faces

\begin{tabular}{ccc}
\hline Pose & $\begin{array}{c}\text { Proposed } \\
\text { method }\end{array}$ & $\begin{array}{c}\text { Non-Histogram } \\
\text { Equalization }\end{array}$ \\
\hline 0 & $95.79 \%$ & $85.31 \%$ \\
1 & $87.34 \%$ & $91.67 \%$ \\
2 & $96.69 \%$ & $90.42 \%$ \\
3 & $80.50 \%$ & $65.93 \%$ \\
4 & $94.21 \%$ & $51.75 \%$ \\
Total & $91.77 \%$ & $82.35 \%$ \\
\hline
\end{tabular}

Finally, hand gesture recognition is conducted on 102 samples of 6 members of our laboratory in the normal condition. We have obtained $92.15 \%$ accuracy in correct recognition.

\section{Conclusion}

In this paper, we proposed a new viewing control method on the robot for comics using face pose and hand gesture recognition. Our face pose recognition method is based on the face pose appearance manifold which is approximated by lowdimensional connected affine plane. Face pose is estimated by computing minimal distance from the given image to the face sub-pose manifold. For robust face pose recognition, we used an occlusion mask for partially occluded faces and a coarse illumination normalization method with histogram equalization for skin-like regions. Empirical evaluation on image sequences with 6 subjects has shown that the proposed method is successful in face pose recognition across illumination and partial occlusion. Our hand gesture method also shows good performance in manipulating object in the panel of the comics.

Our proposed system works at near real-time and shows desirable results under several real situations. Using our proposed system, a user can view comics in his own style. It is worth noticing that our proposed method provides various viewing methods for reading comics through the robots.

The main direction for future work is to handle large head-pose variations and severe illumination variations to facilitate more natural user interaction with consumer devices. Finally, it may prove beneficial to incorporate on-line learning scheme in constructing face pose appearance manifold.

Acknowledgments. This work was supported by the Culture Research Center Project, the Ministry of Culture \& Tourism and the KOCCA R\&D program in Korea.

\section{References}

1. Pentland, A., Moghaddam, B., Starner, B.: View-based and modular eigenspaces for face recognition. In: Proc. IEEE Conf. CVPR, IEEE Computer Society Press, Los Alamitos (1994)

2. Moghaddam, B., Pentland, A.: Probabilistic visual learning for object recognition. IEEE Trans. PAMI (1997) 
3. Moghaddam, B.: Principal manifold and probabilistic subspaces for visual recognition. IEEE Trans. PAMI (2002)

4. Lee, K., Ho, J., Yang, M., Kriegman, D.: Video-based face recognition using probabilistic appearance manifolds. In: Proc. IEEE Conf. CVPR, IEEE Computer Society Press, Los Alamitos (2003)

5. Lee, K., Kriegman, D.: Online learning of probabilistic appearance manifold for videobased recognition and tracking. In: Proc. IEEE Conf. CVPR, IEEE Computer Society Press, Los Alamitos (2005)

6. Ju, M., Kang, H.-B.: A new partially occluded face pose recognition. In: ACIVS 2007. LNCS, vol. 4678, Springer, Heidelberg (2007)

7. Davis, J., Shah, M.: Visual Gesture Recognition. IEE Proc. Vis. Image Signal Processing 141(2) (1994)

8. Chang, C., Chen, I., Huang, Y.: Hand Pose Recognition Using Curvature Scale Space. IEEE ICPR (2002)

9. Hasanuzzaman, Md., Zhang, T., Amporanaramveth, V., Bhuiyan, M., Shirai, Y., Ueno, H.: Face and gesture recognition using subspace method for human-robot interaction. In: Aizawa, K., Nakamura, Y., Satoh, S. (eds.) PCM 2004. LNCS, vol. 3331, Springer, Heidelberg (2004)

10. Malima, A., Ozgur, E., Cetin, M.: A Fast algorithm for vision-based hand gesture recognition for robot control. In: Proc. IEEE Conf. Signal Proc. And Comm. Applications (April 2006)

11. Arandjelovic, O., Shakhnarovich, G., Fisher, J., Cipolla, R., Darrell, T.: Face recognition with image sets using manifold density divergence. In: Proc. IEEE Conf. CVPR, IEEE Computer Society Press, Los Alamitos (2005)

12. Evolution robotics, http://www.evolution.com

13. Viola, P., Jones, M.: Rapid object detection using a boosted cascade of simple features. In: Proc. CVPR (2001)

14. ISO/IEC 14772-1, The Virtual Reality Modeling Language (VRML) (1997), http://www.vrml.org/specifications/VRML97 